\title{
Parallel magnetotransport in multiple quantum well structures
}

\author{
E.M. Sheregii, D. Ploch, M. Marchewka, and G. Tomaka \\ Institute of Physics, University of Rzeszów, Rejtana 16a, 35-310 Rzeszów, Poland \\ E-mail: sheregii@atena.univ.rzeszow.pl \\ A. Kolek, A. Stadler, and K. Mleczko \\ Department of Fundamental Electronics, Rzeszów University of Technology \\ 35-959 Rzeszów, W. Pola 2, Poland \\ W. Strupiński, A. Jasik, and R. Jakiela \\ Institute of Electronic Materials Technology, Wólczyńska 133, 01-919 Warsaw, Poland
}

Received July 21, 2004

\begin{abstract}
The results of investigations of parallel magnetotransport in AlGaAs/GaAs and InGaAs/InAlAs/InP multiple quantum wells structures (MQW's) are presented in this paper. The MQW's were obtained by metalorganic vapour phase epitaxy with different shapes of QW, numbers of $\mathrm{QW}$ and levels of doping. The magnetotransport measurements were performed in wide region of temperatures $(0.5-300 \mathrm{~K}$ ) and at high magnetic fields up to $30 \mathrm{~T}$ ( $\mathrm{B}$ is perpendicular and current is parallel to the plane of the QW). Three types of observed effects are analyzed: quantum Hall effect and Shubnikov-de Haas oscillations at low temperatures $(0.5-6 \mathrm{~K})$ as well as magnetophonon resonance at higher temperatures $(77-300 \mathrm{~K})$.
\end{abstract}

PACS: $73.40 . \mathrm{Hm}$

\section{Introduction}

Electron magnetotransport is widely investigated in low-dimensional structures (LDS) from 1980 year. We can distinguish two type of magnetotransport in LDS according to direction of magnetic field: first magnetic field is perpendicular to plane of quasi-two-dimensional electron gas (Q2DEG) and parallel to $\mathbf{z}$ axis which is axis of the layer growth $(\mathbf{B} \| \mathbf{z})$ and second - magnetic field is parallel to plane of Q2DEG and perpendicular to $\mathbf{z}$ axis $(\mathbf{B} \perp \mathbf{z})$. In each of these cases both perpendicular electron transport (current is perpendicular to plane of Q2DEG) and parallel electron transport (current is parallel to plane of Q2DEG) are possible. It is well known that the Landau quantization occurs in plane perpendicular to magnetic field. If magnetic field $\mathbf{B}$ is perpendicular to plane of Q2DEG or parallel to $\mathbf{z}$ axis complete value of $B$ caused the Landau quantization which means oscillator character of density of states. The quantum effects in Q2DEG as Shubnikov - de Haas oscillations
$(\mathrm{SdH})$ and quantum Hall effects (IQH) are caused by Landau quantization and can be observed in case of perpendicular to layer magnetic field $(\mathbf{B} \perp \mathbf{z})$ and parallel electron transport.

If $\mathbf{B}$ is not perpendicular to plane of Q2DEG and there is angle $\varphi$ between $\mathbf{B}$ and $\mathbf{z}$, the Landau level energy is formed by value $B_{t}=B \cos \varphi$. It is well known effect of tilted magnetic field when the resonance peaks are shifted toward strong magnetic fields if $\mathbf{B}$ is tilted to plane of Q2DEG (angle $\varphi$ increase).

Any Landau quantization we will observe if $\mathbf{B}$ is located in plane of Q2DEG. The barrier effects can be appeared only in perpendicular electron transport in this case for Q2DEG in heterostructures or single quantum well (QW). The interesting effects are manifested for this geometry if we have coupled double QW (DQW): in DQW will be appeared effect connected with crossing of Fermi surfaces.

Both perpendicular and parallel electron transports have very important applications. 
Firstly: perpendicular electron transport has fundamental application, namely - lasers as well as detectors of electro-magnetic radiation. The determination of the Q2DEG parameters in conditions of perpendicular transport is one of more important aim of the perpendicular electron magnetotransport research.

Secondly: parallel electron transport has fundamental application too, namely transistors and first of all - special transistors as i) high power transistors, ii) transistors for high frequency or high electron mobility transistors (HEMT). The conditions for such devices are fulfilled in GaAs/AlGaAs 2D structures produced by MBE technology, as well as MOVPE.

The strained quantum wells such as the InGaAs / InP (InGaAs / InAlAs) structures are widely used for the high-speed semiconductor devices, such as InGaAs high-speed transistors or HEMT [1] and heterojunction bipolar transistors (HBT) [2-4]. Transistors built on base of InGaAs/InAlAs structures with single QW have higher frequency limit and lower noise in comparison with one performed on GaAs/AlGaAs. But the last structures have very essential advantage - they can be heated to higher temperature without deterioration of parameters what is important for high power devices. So, it is paramount for HEMT based on InGaAs / InAlAs structures to obtain wider QW with sufficient high electron density (lower resistance). From other hand the wide QW means appearing of the three-angular shape of QW's where is located Q2DEG which make impossible increasing of electron density without deterioration of the carrier parameters. The special technology of HEMT structures is required to obtain the wide quasirectangular QW with Q2DEG of high parameters $[5,6]$. Therefore, the HEMT creation built on InGaAs/InAlAs/InP QW structures of different shape is problem of considerable importance. The separation of donors from carriers in $\mathrm{QW}$ by space buffer enables us to decrease the their scattering. The direct doping of QW is applied for power amplifiers aimed to decrease amplitudes of harmonics in signal [3].

The LDS's can contain different number of QW's:

- two QW named as double quantum wells (DQW);

- several identical (or not) QW we will call as multiple quantum wells (MQW);

- great number of identical QW are named as superlattices (SL).

The HEMT can be built on MQW too because second or more then two channels increase the conductivity and the device power limit. Therefore, research of the electron parallel transport in channels of the MQW structures GaAs / AlGaAS and InGaAs / InP or InGaAs/InAlAs/InP is interesting from point of view an improvement of the $2 \mathrm{DEG}$ parameters.

\section{The single QW (SQW) structures}

Investigations of structures with single QW are important to get experience in interpretation of quantum effects: SdH oscillations and IQH. It is necessary to develop methods of calculation of the Landau level energies in SQW of different shape: rectangular and triangular. These methods must be verified on real QW's. The detail description of the engineering of structures as well as describing of the experimental data and their interpretation are presented in original articles $[7,8]$. Here we present the main results only.

\subsection{Structures}

Structures InGaAs/InAlAs/InP with single QW were grown by low pressure metalorganic vapour phase epitaxy (LP-MOVPE) on semi-insulating (100) InP:Fe substrates. A horizontal quartz reactor (AIX-200R\&D) and IR heated graphite susceptor were used. The reactor pressure and temperature were maintained at $100 \mathrm{mbar}$ and at $650^{\circ} \mathrm{C}$, respectively, during the unstrained layer growth. The $\mathrm{V} / \mathrm{III}$ ratio was unchanged and amounted to 329 for $\mathrm{InP}, 1040$ for $\mathrm{In}_{0.52} \mathrm{Al}_{0.48} \mathrm{As}$ and 198 for $\mathrm{In}_{0.75} \mathrm{Ga}_{0.25}$ As. The partial pressure of $\mathrm{SiH} 4$ (doped barriers) was this same in all of structures.

Four types of structures were studied. Their parameters are presented in Table 1. The first structure (\#1093) consisted of (from bottom to the top) an undoped $180 \mathrm{~nm}$ InP buffer followed by $400 \mathrm{~nm}$ InAlAs buffer layer on InP:Fe substrate. A delta-doping Si donor layer and $3 \mathrm{~nm} \mathrm{In} 0 .{ }_{2} \mathrm{Al}_{0.48} \mathrm{As}$ spacer were grown on InAlAs buffer layer. An $13 \mathrm{~nm} \mathrm{In}{ }_{0.75} \mathrm{Ga}_{0.25}$ As channel layer, $3 \mathrm{~nm} \operatorname{In}_{0.52} \mathrm{Al}_{0.48} \mathrm{As}$ spacer, delta-doping Si donor layer and $25 \mathrm{~nm} \mathrm{In}{ }_{0.52} \mathrm{Al}_{0.48} \mathrm{As}$ Schottky layer terminated the structure growth.

Table 1

Parameters of structures

\begin{tabular}{|c|c|c|c|c|}
\hline \multirow[t]{2}{*}{ Sample } & \multicolumn{3}{|c|}{ Channel parameters } & \multirow{2}{*}{$\begin{array}{c}\delta \text {-doping do- } \\
\text { nor concen- } \\
\text { trations, } \\
10^{12} \mathrm{~cm}^{-2} \\
\end{array}$} \\
\hline & $\begin{array}{l}\text { composi- }^{-} \\
\text {tions, In, \% }\end{array}$ & $\begin{array}{l}\text { thick- } \\
\text { ness, nm }\end{array}$ & profile of QW & \\
\hline 1093 & 75 & 20 & sharp interface & 0.7 \\
\hline 1098 & 65 & 20 & $\begin{array}{l}\text { changed com- } \\
\text { positions in } \\
\text { channel }\end{array}$ & 5.0 \\
\hline 1607 & 65 & 23.5 & sharp interface & 3.5 \\
\hline $1088^{*}$ & 53 & 20 & sharp interface & 2.5 \\
\hline
\end{tabular}

Comment: $1088^{*}-$ process with one $\delta$-doping layer.

In the case of the second structure (\#1098), the In-content in the channel was changing from 53 to $65 \%$ during the time of $25 \mathrm{~s}$, then In-content of $65 \%$ in 
the $17 \mathrm{~nm}$ InGaAs layer was kept constant and graded layer (from 65 to 53\%) was repeated. Parameters of the others layers were unchanged.

Third (\#1088) structure has only one delta-doping Si donor layer, in comparison with the previous structures. The 10 period: $(1.8 \mathrm{~nm}) \quad \mathrm{In}_{0.53} \mathrm{Ga}_{0.47} \mathrm{As} /(2.5 . \mathrm{nm})$ $\mathrm{In}_{0.52} \mathrm{Al}_{0.48} \mathrm{As}$ superlattice were deposited in case of \#1607 as the buffer on InP layer, additionally. In order to improve the crystalline quality of the strained layer, the growth rate and temperature values of the $18 \mathrm{~nm} \mathrm{In}{ }_{0.65} \mathrm{Ga}_{0.35}$ As active layer were lower than the ones in the case of \#1098 structure and equal to $4.1 \AA / \mathrm{s}$ and $600^{\circ} \mathrm{C}$, respectively.

The structures \#1093, \#1607 and \#1088 have sharp interfaces between the InGaAs channel layer and the InAlAs spacer layers. The doping level was different in this four kind of structures: minimal for \#1093 and maximal for \#1098.

\subsection{Description of experiment and results}

Magnetotransport measurements were performed with using superconducting system giving magnetic fields up to $11 \mathrm{~T}$. The sample was mounted in anticryostat providing temperatures from 0.4 to $300 \mathrm{~K}$. The $U_{x y}(B)$ and $U_{x x}(B)$ records registered for opposite direction of magnetic and electric fields. Therefore, eight records were made and averaged at one temperature measurement for both Hall effect and magnetoresistance.

In Fig. 1 and 2 are shown examples of $R_{x y}(B)$ and $R_{x x}(B)$ records for samples \#1088, \#1607 and \#1098.

The formation of Q2DEG in the samples \#1088, \#1607 and \#1098 is clearly evidenced by quantum oscillations in $R_{x x}(B)$ and plateaux in $R_{x y}(B)-\mathrm{IQH}$. It is shown that Hall resistance $R_{x y}(B)$ at temperature $0.4 \mathrm{~K}(0.6 \mathrm{~K}), 1.6 \mathrm{~K}$ and $2.8 \mathrm{~K}$ is quantized according to $h / v e^{2}$ where $v$ is an integer, $h$ is the Planck constant and $e$ is the electron charge (in case of the sample \#1607 there is a departure from this rule, discussed later). The pronounce maxima of the $R_{x x}(B)$ curve (the $\mathrm{SdH}$ oscillations) correspond to beginning of each plateau on the $R_{x y}(B)$ curve. These oscillation peaks decline at temperatures upper $10 \mathrm{~K}$. It is necessary to notice that structure of the $\mathrm{SdH}$ peaks in case of the sample \#1088 is simpler and more clarity in comparison with oscillations peaks for the sample $\# 1607$. At the temperature of $0.4 \mathrm{~K}$, for the sample $\# 1607$ there is a long plateau on the curve $R_{x y}(B)$, which spreads from 4.0 to $6.5 \mathrm{~T}$ with the maximum at magnetic field of about $8.0 \mathrm{~T}$, where the $v=5$ plateau should start of (this maximum disappeared at $T>1.0 \mathrm{~K}$ and the $v=5$ plateau becomes to be observable). While, for the sample \#1098 at $T=0.6 \mathrm{~K}$ the sequence of pronounce plateaux on the $R_{x y}(B)$ curve are ob-
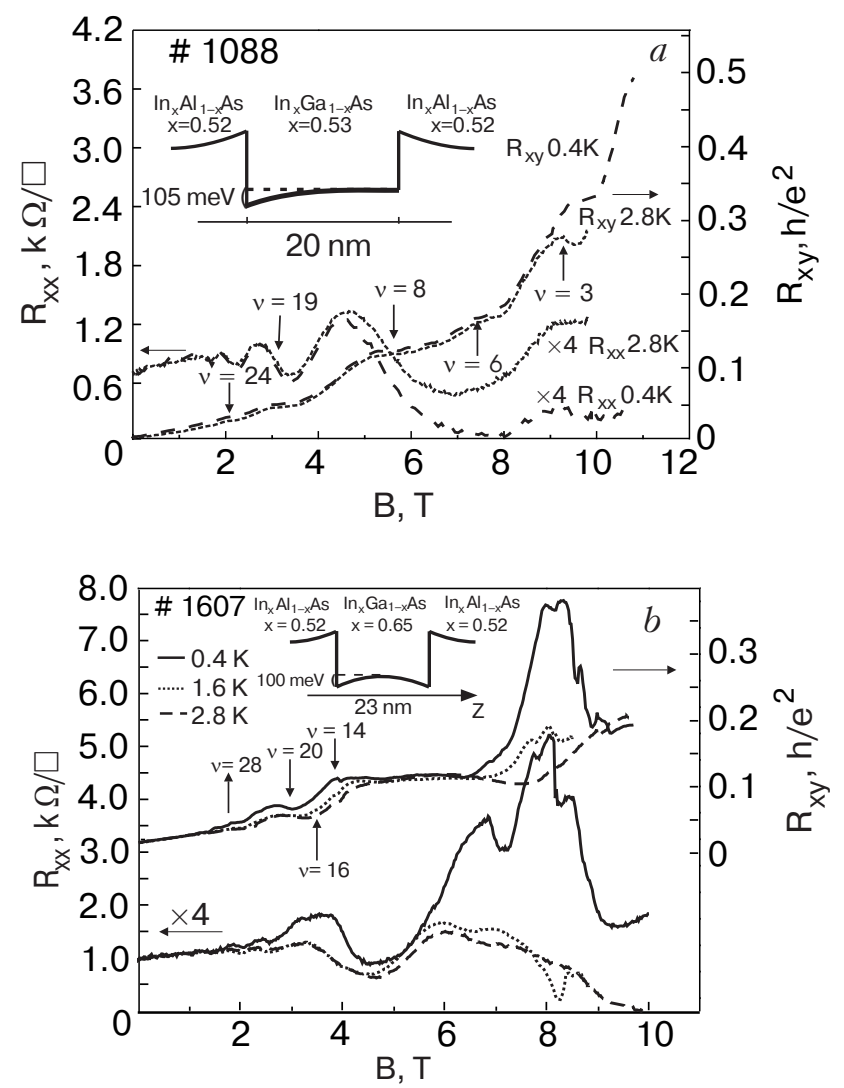

Fig. 1. The records of the $R_{x y}(B)$ and $R_{x x}(B)$ for samples \#1088 $(a)$ and \#1607 $(b)$. The inset shows the band profile of the QW.

served. There are also observed very sharp maxima on the $R_{x x}(B)$ curve with undoubtedly periodic behavior versus inverse magnetic field.

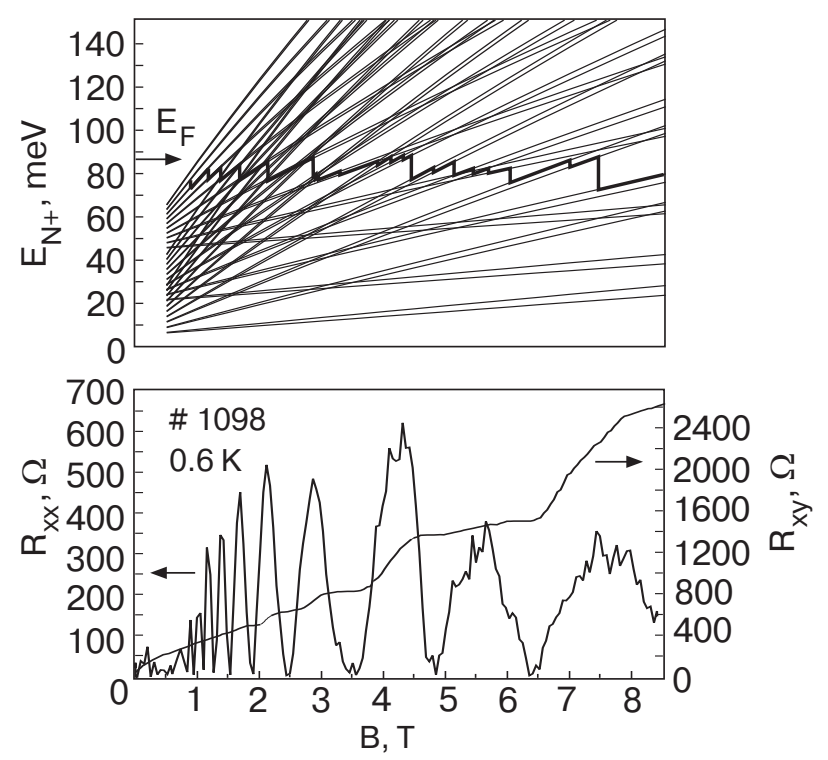

Fig. 2. The Landau levels energies calculated for sample \#1098 as well as SdH oscillations and IQH at 0.6 K. 


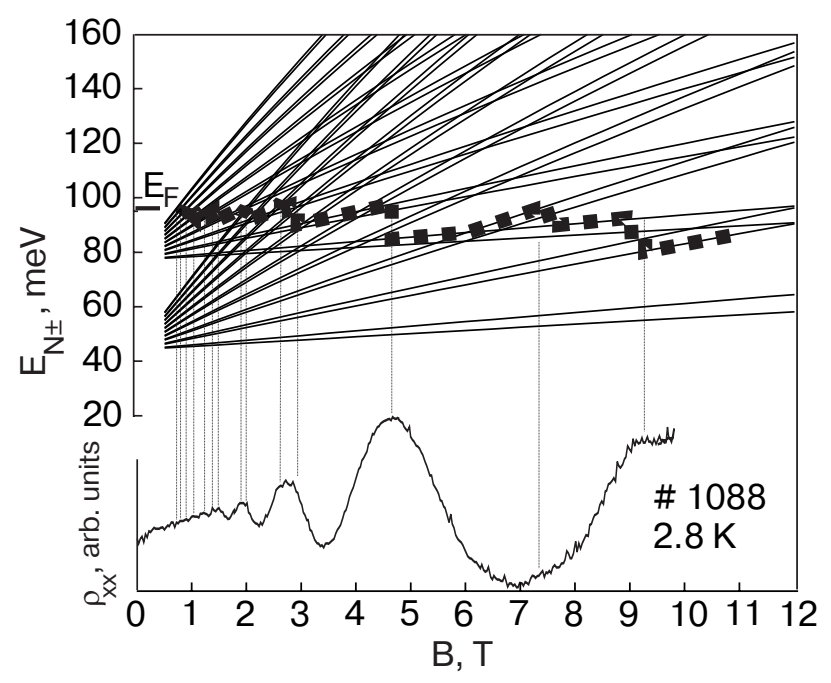

Fig. 3. The Landau levels energies calculated for sample \#1088 and SdH oscillations at $2.8 \mathrm{~K}$.

Analysis of positions of experimental peaks in inverse magnetic field shows presence more than one oscillation series: two for the sample \#1088, and three for the sample \#1098 (without spin splitting). That means population of two subbands in QW in cases of the samples \#1088 and three subbands in case of the sample \#1098. Another confirmation that the two electron subbands for the samples \#1088 are populated as well as the three subbands for the sample \#1098 is deduced from the IQH filling factors $v$ and the Landau levels energies calculation (see Fig. 3 and 4). Similar analysis for the sample \#1607 was done by means $R_{x x}(B)$ and $R_{x y}(B)$ curves obtained at $T=1.6 \mathrm{~K}$.

The fundamental fields $B_{f}$ for this series are presented in Table 2. Assuming a circular Fermi surface, a

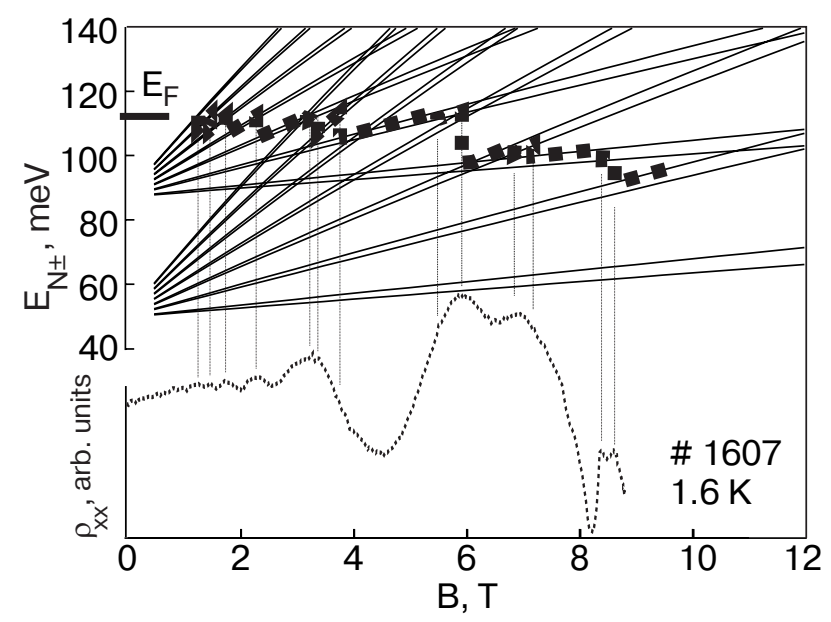

Fig. 4. The Landau levels energies calculated for sample \#1607 and SdH oscillations at $1.6 \mathrm{~K}$.
2D $n_{s}$ concentration of carriers can be extracted from the $\mathrm{SdH}$ period:

$$
n_{s}=\sum_{i}^{k} \frac{2 e\left(B_{f}\right)_{i}}{h},
$$

where $i$ is number of subband in QW and $k$ is the number of populated subbands. The magnitudes of density $n_{s}$ are presented in Table 2 . The values of carrier's mobility determined from low-field Hall measurements are placed here too.

In case of the sample \#1093 the oscillation peaks on the $R_{x x}(B)$ curve are observed too but they are not so pronounce as for sample described above and the plateaux on the $R_{x y}(B)$ curve are absence.

Table 2

The values of fundamental fields $B_{f}$ of $\mathrm{SdH}$ oscillations and $2 \mathrm{D}$ carrier density deduced from $B_{f}$ as well as the carrier mobilities obtained from $\mathrm{SdH}$ oscillations dampfing factor

\begin{tabular}{|c|c|c|c|c|c|}
\hline \multirow[t]{3}{*}{ Sample } & \multicolumn{3}{|c|}{$B_{f}, \mathrm{~T}$} & \multirow{3}{*}{$\begin{array}{l}\text { 2D carrier } \\
\text { density, } \\
10^{12} \mathrm{~cm}^{-2}\end{array}$} & \multirow{3}{*}{$\begin{array}{c}\text { Electron } \\
\text { mobility }^{*} \\
10^{5} \mathrm{~cm}^{2} /(\mathrm{V} \cdot \mathrm{s})\end{array}$} \\
\hline & \multicolumn{3}{|c|}{$\begin{array}{c}\text { Number of electronic } \\
\text { subbands }\end{array}$} & & \\
\hline & 1 & 2 & 3 & & \\
\hline 1088 & 2.6 & 19.6 & - & 1.07 & 1.7 \\
\hline $1607^{* *}$ & 6.4 & 19.8 & - & 1.2 & 3.2 \\
\hline 1098 & 8.3 & 14.9 & 18.4 & 2.0 & 2.6 \\
\hline 1093 & 6.1 & 19.4 & - & 1.0 & 0.94 \\
\hline
\end{tabular}

Comments: ${ }^{*}$ is related to Landau levels in the magnetic field region about $1.5-4 \mathrm{~T}$; ** parameters are related to temperatures $T>1.0 \mathrm{~K}$.

\subsection{Interpretation}

In case of the sample \#1088 we have asymmetric $\mathrm{QW}$. The band profiles of this QW were calculate by Zawadski and Pfeffer [9] (the composition of barrier is different only of $1 \%$ ) and are presented in Fig. 1,a (inset). Since, the deep of triangular well in edge of QW for structure \#1088 is $105 \mathrm{meV}$ and we can assume that the Q2DEG in this structure is placed in single triangular potential well. The structure \#1607 was doped symmetrically, since shape of $\mathrm{QW}$ is symmetric too. This shape is show in Fig. 1,b (inset). In case of sample \#1607 we have two triangular QW deviated by barrier of about $95 \mathrm{meV}$. So, the Q2DEG in structure \#1607 is located in nearing double triangular QW.

The engineering of the structure \#1098 is more complicated: the In-content in the channel increase from 53 to $65 \%$ during the $16 \mathrm{~nm}$ to compensate the Coulomb interaction with ionized donors in $\delta$-doping 
layers. Therefore, the shape of QW is practically rectangular (see Fig. 5).

To interpret experimental results on the $\mathrm{SdH}$ oscillations and IQH plateaux it is necessary to calculate energy of Landau levels. We adapted Zawadzki-Pfeffer quazi-two-band model $[10,11]$ for calculation of the Landau level energies in subbands of QW. The description of the adapted model for the different shape QW is presented in [8].

The calculated Landau levels are shown in Fig. 3 for sample \#1088 (triangle QW) at temperature $2.8 \mathrm{~K}$. The structure of the $\mathrm{SdH}$ peaks as well as the plateaux of the IQH effect are interpreted very well for this sample: it is necessary to take the value of Fermi energy equal to $18 \mathrm{meV}$ for subband of number $i=1$ and $51 \mathrm{meV}$ for subband with $i=0$ (we define the Fermi level us to be placed between two Landau levels when the plateaux on the $R_{x y}(B)$ curve or the minima (zero) on the $R_{x x}(B)$ curve occur).

The calculated Landau levels are shown in Fig. 2 for the sample \#1098 (rectangular QW) at temperature $0.6 \mathrm{~K}$. It is necessary to take the value of the Fermi energy equal to $27.5 \mathrm{meV}$ for subband of number $i=2,54 \mathrm{meV}$ for subband with $i=1$, and $70 \mathrm{meV}$ for the lowest subband with $i=0$ to obtain good agreement with positions in magnetic fields of experimental peaks of the SdH oscillations for the sample \#1098.

In case of the sample \#1607 the satisfactory agreement between experimental positions of the $\mathrm{SdH}$ peaks versus magnetic fields and theoretical ones at $T=1.6 \mathrm{~K}$ was obtained if the Landau level energies were calculated in the framework of a single triangle QW model. The values of the Fermi level energies are 27 and $63 \mathrm{meV}$ for subbands with $i=1$ and $i=2$, respectively. But this model can not be adjusted for the $R_{x y}(B)$ and $R_{x x}(B)$ curves obtained at $0.4 \mathrm{~K}$.

The values of the band-structure parameters, energy gap $E_{g}$, the effective electron mass on edge of conduction band $m_{c}^{*}$, effective $g_{c}^{*}$ factor used for calcu-

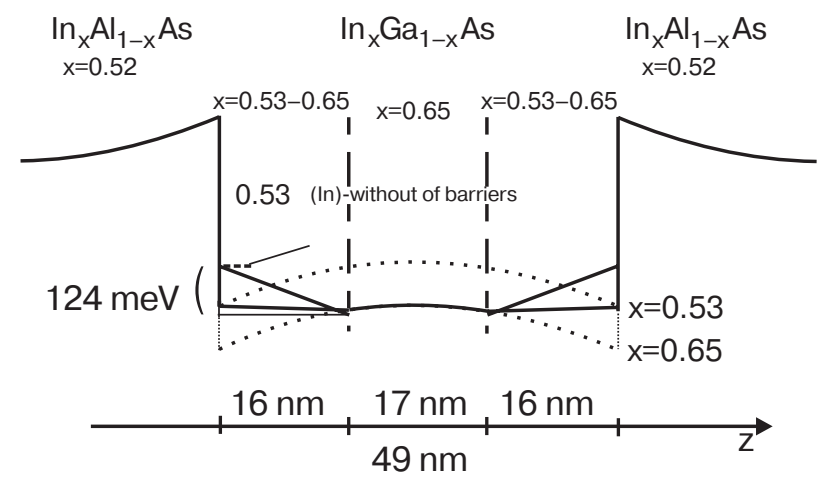

Fig. 5. The band profile of the QW for structure \#1098. lation the Landau levels energies of Q2DEG for all structures are presented in Table 3.

Table 3

Band-structure parameters of $\operatorname{In}_{x} \mathrm{Ga}_{1-x}$ As channels of the In GaAs / InAlAs / InP structures

\begin{tabular}{c|c|c|c|c|c}
\hline \hline Sample & $x$ & $E_{g}, \mathrm{eV}$ & $m_{c}^{*} / m_{0}$ & $g_{c}^{*}$ & $\Delta, \mathrm{eV}$ \\
\hline \hline 1088 & 0.53 & 0.846 & 0.0345 & -8.16 & 0.355 \\
1607 & 0.65 & 0.723 & 0.0299 & -9.90 & 0.355 \\
1098 & 0.65 & 0.723 & 0.0299 & -9.90 & 0.355 \\
1093 & 0.75 & 0.629 & 0.0265 & -11.36 & 0.355 \\
\hline \hline
\end{tabular}

\subsection{Generalization}

There were prepared four kind InGaAs / InAlAs / InP structures with SQW channel for HEMT: with sharp interface - the structures \#1088, \#1607 and \#1093 and with changed composition in channel - the \#1098 structure.

Structures with sharp interface have different content of InAs in QW channel: $53 \%$ for \#1088, 65\% for $\# 1607$, and $75 \%$ for \#1093. It is shown that maximal mobility of Q2DEG in the InGaAs QW channel was obtained for the structure \#1088 with single asymmetric triangle $\mathrm{QW}$. The lowest electron mobility was determined for the structure \#1093 with maximal difference of the InAs content in barrier and QW, namely: 53 and $75 \%$, respectively, though the effective mass of electrons in QW is the smallest. That means that scattering on the interface dislocations is significant and we can assume that this factor is most important in limitation of the electron mobility in QW.

The interesting case represent the structure \#1098 where was prepared the smooth transition from barrier to QW with aim to decrease mismatch as well as to form rectangular $\mathrm{QW}$. The calculations confirm that determination of the Landau level energies by model applied for the rectangular QW give better fitting to experimental positions of the $\mathrm{SdH}$ peaks then model applied to the triangle QW. So, the InGaAs channel in the structure \#1098 really forms the quasi-rectangular QW. The 2D density of electrons in this channel is the highest and the electron mobility is very high too, namely $2.6 \cdot 10^{5} \mathrm{~cm}^{2} /(\mathrm{V} \cdot \mathrm{s})$. Therefore, the proposed engineering of this structure seems to be perspective.

The Zawadzki - Pfeffer quazi-two-band model [10,11] adapted for calculation of the Landau level energies in the triangle and rectangular InGaAs/InAlAs QW enables us satisfactorily to interpret experimental data for the structures \#1088 and \#1098. 


\section{Double quantum well}

\subsection{Motivation}

Fabrication of multiple 2D layers in close proximity allows the controlled introduction an additional degree of freedom associated with third dimension. The double QW is the simplest of these structures and preserves both high electron mobility and external gating of electron density in the layers. Interlayer Coulomb interaction in multilayer systems can lead to interesting effects such as Wigner crystals or loose of plateaux in IQH for filling factor equal to 1 or 3 [12,13]. Experimentally both integral and fractional Hall effects were observed in multilayer systems with essential interlayer tunnelling. Effects connected to the crossing of the Fermi surfaces of two coupling QW when $B$ there is in plane of layers were observed for wide QW with Q2DEG localized in two triangle QW [14] or for specially engineered DQW [15]. Problem of the electron-electron interaction for coupled DQW was investigated experimentally in Refs. 16, 17 and theoretically by Danhong Huang et al. [18]. So, bi-layer two-dimensional systems have been of great research interest in the studying of the properties of Q2DEG under the strong magnetic fields.

\subsection{The structure description and experimental results}

Here the experimental results are presented which were obtained for DQW fabricated in the Institute of Electronic Material Technology in Warsaw. They consisted of two InGaAs QW of $20 \mathrm{~nm}$ thickness and three InAlAs barriers. In each barrier there were the donor $\delta$-doping layers.

The structure was grown up on InP substrate similarly to SQW. Parameters of the structure are present in Table 4. The data of magnetotransport measurements at low temperatures are shown in Fig. 6, where the pronounced $\mathrm{SdH}$ oscillations on the $R_{x x}(B)$ curve as well as the IQH effect on the $R_{x y}(B)$ curve of are shown also. The $\mathrm{SdH}$ oscillations for weak magnetic fields appear from $0.5 \mathrm{~T}$. The peaks of the $\mathrm{SdH}$ oscillations at higher magnetic fields are splitted just like it was observed in DQW by other authors.

This splitting increases towards higher magnetic field which means the energy gap caused by electron

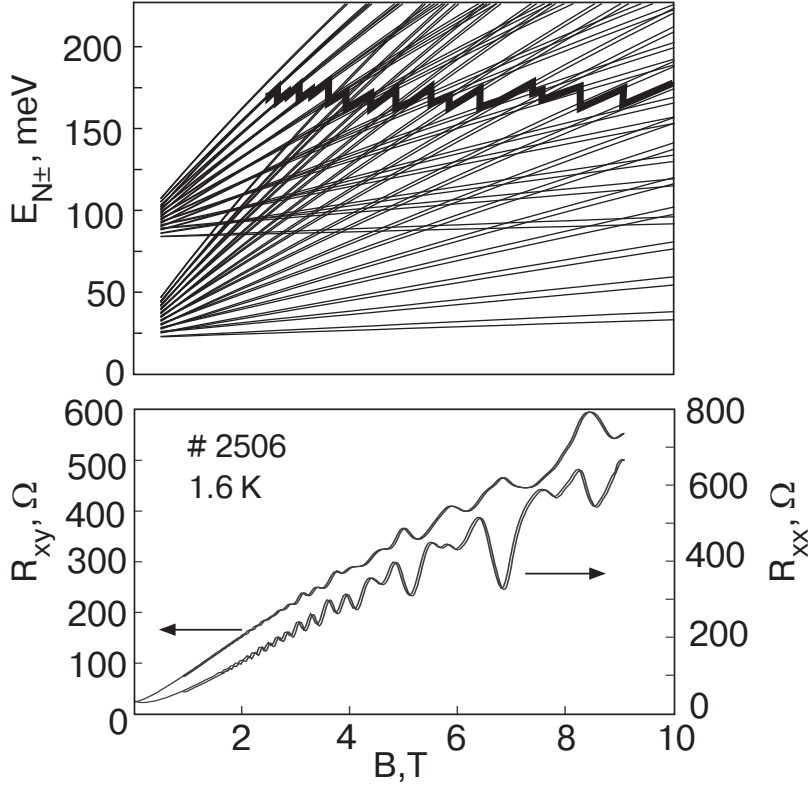

Fig. 6. The Landau levels energies calculated for sample \#2506 as well as SdH oscillations and IQH at $1.6 \mathrm{~K}$.

interaction within DQW's is proportional to $B$ as it was shown in Ref. 16. It is necessary to notice that the plateaus on the $R_{x y}(B)$ curve are deformed. There are two possible explanations of that. The first explanation refers to the presence of additional channels of conduction, for instance conduction through three donor $\delta$-layers. The second one refers to the QW's coupling.

\subsection{Theory}

We use the model proposed in Ref. 18, in which the symmetric DQWs separated by the middle barrier, were considered. An external magnetic field was considered to be applied perpendicular to the planes of QW's. The electron motion was described by the Schrödinger equation containing both the DQW potential and self-consistent Hartree potentials. The in-plane electron motion in each subband in the DQW was supposed to be Landau quantized. The total electron energy including both the in-plane and vertical electron motions can be written as

$$
E_{n i}=\left(n+\frac{1}{2}\right) \hbar \omega_{c}+E_{i}+V_{n i}^{F}
$$

where $V_{n i}^{F}$ is the exchange energy (involving screening) which is given by Eqs. (12)-(18) in Ref. 18. The

Table 4

Parameters of the InGaAs / InAlAs DQW structure

\begin{tabular}{|c|c|c|c|c|}
\hline Sample & Composition of two channels In, \% & Two channels with thickness of, $\mathrm{nm}$ & QW-profile & $\begin{array}{l}\text { Three } \delta \text {-doping layers with donor } \\
\text { concentrations, } 10^{12} \mathrm{~cm}^{-2}\end{array}$ \\
\hline 2506 & 65 & 20 & Sharp interface & 3.5 \\
\hline
\end{tabular}


numerical calculations performed by the authors of [18] for DQW based on the well material GaAs and barrier material $\mathrm{Al}_{0.3} \mathrm{Ga}_{0.7}$ As with the well wide of $14 \mathrm{~nm}$ and the barrier wide of $3 \mathrm{~nm}$, have shown that there is an approximately linear $B$ dependence for the tunnelling gap in the region of magnetic fields $B \leq 9 \mathrm{~T}$. Therefore, the total splitting $\Delta_{t}$ between energy states of two QW's consists of two parts: constant $\Delta_{S A S}$, determined by the overlapping of the electron wave functions of two QW's and changeable tunnelling term $V_{n i}^{F}$, involving screening factor:

$$
\Delta_{t}=\Delta_{S A S}+V_{n i}^{F} \text {. }
$$

The last term is strong reducing with increase in $B$ and for magnetic field less then that one, for which quantum limit begins, can be written as

$$
V_{n i}^{F}=-\left(K_{0}-k B\right) \text {, }
$$

where $K_{0}$ is the maximum screening factor in zero magnetic field and $-k B$ is changeable screening factor decreasing with $B$ up to first Landau level in two QW's were filled. Generally, the total energy gap $\Delta_{t}$ between symmetric and antisymmetric states in DQW is proportional to the magnetic field as it was observed experimentally [16].

We adapted this consideration for our case of $\mathrm{In}_{0.53} \mathrm{Al}_{0.47} \mathrm{As} / \mathrm{In}_{0.65} \mathrm{Ga}_{0.35} \mathrm{As}$ DQW structure assuming rectangularity of QW's. The last one enables us to calculate the Landau level energies according to the formulae:

$$
\begin{gathered}
\frac{\left(E-E_{\perp}\right)\left(E_{g}+E+E_{\perp}\right)}{E_{g}}=\frac{\hbar^{2} \pi^{2}(i+1)^{2}}{2 m_{c}^{*} a^{2} k} \pm \\
\pm\left(\Delta_{S A S}^{s}+k E_{\perp}\right),
\end{gathered}
$$

$E_{\perp}=-\frac{E_{g}}{2}+\frac{E_{g}}{2} \sqrt{1+\frac{4 \mu_{B} B}{E_{g}}\left[f_{1} \frac{m_{0}}{m_{c}^{*}}\left(n+\frac{1}{2}\right) \pm \frac{1}{2} g_{0}^{*} f_{2}\right]}$,

$$
\begin{gathered}
f_{1}=\frac{\left(E_{g}+\Delta\right)\left(E_{\perp}+E_{g}+\frac{2}{3} \Delta\right)}{\left(E_{g}+\frac{2}{3} \Delta\right)\left(E_{\perp}+E_{g}+\Delta\right)}, \\
f_{2}=\frac{E_{g}+\frac{2}{3} \Delta}{E_{\perp}+E_{g}+\Delta},
\end{gathered}
$$

where $E_{g}$ is energy gap (between the top of valence band and the edge of conduction band) and $\Delta$ is spin-orbital splitting, $m_{0}$ is electron mass in vacuum, $m_{c}^{*}$ is the effective electron mass on the edge of the conduction band, $\mu_{B}$ is the Bohr magneton, $n=$ $=0,1,2, \ldots$ stands for Landau levels, $i=0,1,2, \ldots$ is the number of subbands, $E_{\perp}$ is the Landau level energy in the absence of QW (in Zawadzki - Pfeffer notation) and $E$ is unknown energy of Landau level $n$ for subband with number $i$ in QW. Parameter $\Delta_{S A S}^{s}$ is the energy gap determined by overlapping of the wave functions which is diminished by the screening at zero magnetic field as follow on from (3) and (4):

$$
\Delta_{t}=\left(\Delta_{S A S}-K_{0}\right)+k B \equiv \Delta_{S A S}^{S}+k B \text {. }
$$

This parameter means the zero magnetic field splitting of subbands and can be determined by extrapolation of the experimental peaks splitting of the $\mathrm{SdH}$ oscillations to $B=0$. The parameter $k$ (in fact, it is the coefficient of proportionality between the total splitting $\Delta_{t}$ and $\hbar \omega_{c}$ ) can be determined as fitting parameter.

\subsection{Interpretation}

The calculation of the Landau level energies was performed for the sample \#2506 taking into account the band parameters determined for sample \#1098 with single rectangular QW (Table 3). The extrapolation of the experimental peaks splitting of the $\mathrm{SdH}$ oscillations to $B=0$ gives the value of $\Delta_{S A S}^{S}=1 \mathrm{meV}$. The coefficient of proportionality $k$ between total splitting $\Delta_{t}$ and $\hbar \omega_{c}$ was determined as fitting parameter and occurred to be equal to 0.19 .

In Fig. 6 the calculated Landau level energies are shown as the function of magnetic field. It is clearly seen that we achieved an excellent agreement between the experimental data concerning peak positions and theoretical prediction for the SdH oscillation maxima. It is interesting to note that the value of total splitting $\Delta_{t}$ is approximately equal to $15 \mathrm{meV}$ at $10 \mathrm{~T}$ for $n=1$ and $i=0$ and should increase together with field increasing up to about $30 \mathrm{~T}$, where the predicted quantum limit should occur. It is necessary to notice that we needed not to involve the thermostresses caused by mismatch to explain experimental data on the InGaAs/InAlAs/InP DQW.

If we return back to Fig. 1, $b$ where the experimental data for the sample \#1607 are presented, we can notice that a strong maximum at $8 \mathrm{~T}$ in the $R_{x y}(B)$ curve obtained for this sample at $T=0.4 \mathrm{~K}$ can be explained by the presence of second triangle $\mathrm{QW}$ in this sample. ${ }^{*}$ As it was mentioned above, the $\mathrm{SdH}$ oscilla-

* The maximum in the plateaux with $v=1$ was observed on $R_{x y}(B)$ curve by M. Kellog et al. [17] for DQW and was explained by referring to the drug effect in IQH effect. 
tion structure at $2.8 \mathrm{~K}$ can be explained by means of the single-triangle QW model. This model predicts the value of Fermi energy as to be about $112 \mathrm{meV}$ above the conduction band bottom. This value is a bit greater than the barrier energy height $(\sim 95 \mathrm{meV})$. This barrier separate two triangles $\mathrm{QW}$ in conducting channel and hence, Q2DEG in two triangles QWs makes a united electron system. We assume that at $0.4 \mathrm{~K}$ the Fermi level decrees below the barrier top. Then the new situation occurs when the two correlated electron subsystems are in two separate triangle QWs. Therefore, in this situation the structure should be considered as DQW.

\subsection{Generalization}

In parallel and perpendicular magnetotransport in DQW the effects connected to the resonance tunnelling are appeared:

- the existence of SAS gap which means an additional splitting of electronic states;

- SAS gap is proportional to $B$; this proportionality is expected to be reduced in very strong magnetic field.

There was achieved the excellent agreement between the experimental data concerning the $\mathrm{SdH}$ peak positions and theoretical prediction for the $\mathrm{SdH}$ oscillation maxima based on the method of the Landau level energies calculation involving the model of the DQW proposed in Ref. 18 and the Zawadzki-Pfeffer quazi-two-band model $[10,11]$.

The influence of the thermostresses on parallel electron transport that can disintegrate the magnetoconductivity in the InGaAs/InAlAs DQW, was not observed in this measurement.

\section{Multiple quantum wells}

\subsection{Description of structures}

Four types of MQW systems were studied. They consisted of ten QW of GaAs and ten $\mathrm{Al}_{x} \mathrm{Ga}_{1-x}$ As barriers, and were obtained by the LP-MOVPE on semi-insulating GaAs at Warsaw Institute of Electronic Materials. The thickness of the well was 10 and $8 \mathrm{~nm}$, the thickness of the barrier was $4 \mathrm{~nm}$ for all MQW. GaAs layers were doped with $\mathrm{Si}$ to about $5 \cdot 10^{10}$ and $5 \cdot 10^{11} \mathrm{~cm}^{-3}$. The parameters of MQW's are shown in Table 5.

Six samples in standard form for Hall measurements obtained by photolithography from \#151 MQW's have been measured as well as three, two and two from \#152, \#153 and \#41, respectively.
Parameters of the GaAs $/ \mathrm{Al}_{x} \mathrm{Ga}_{1-x} \mathrm{As} \mathrm{MQW}$ structures

\begin{tabular}{c|c|c|c}
\hline \hline Structures & $\mathrm{L}_{\mathrm{GaAs}}(\mathrm{nm}) / \mathrm{L}_{\mathrm{AlGaAs}}(\mathrm{nm})$ & $x$ & $\begin{array}{c}\text { The carrier den- } \\
\text { sity, } 10^{10} \mathrm{~cm}^{-2}\end{array}$ \\
\hline \hline 151 & $10 / 4$ & 0.3 & 50 \\
152 & $10 / 4$ & 0.3 & 5 \\
153 & $8 / 4$ & 0.3 & 50 \\
41 & $10 / 4$ & 1.0 & 50 \\
\hline \hline
\end{tabular}

\subsection{Experimental results: low-temperature measurements}

The data of magnetotransport measurements at low temperatures are presented in Fig. 7 for the sample \#151. It is shown that there are the $\mathrm{SdH}$ oscillations on the $R_{x x}(B)$ curve. The oscillations peaks start at the magnetic field of about $4.5 \mathrm{~T}$ and consist of three unsplitted maxima. The $\mathrm{SdH}$ oscillations start at higher field than that one in the QW structures based on InGaAs / AlGaAs, because the electron effective mass in GaAs is two times greater than in $\operatorname{In}_{0.65} \mathrm{Ga}_{0.35}$ As. The single plateau is observed on the $R_{x y}(B)$ curve at 8-9 $\mathrm{T}$, it corresponds to the deep minimum on the $R_{x x}(B)$ curve. The similar curves were obtained at low temperatures for the samples \#41 and \#152. These curves, on one hand, is the evidence of the existence of Q2DEG in the samples which we studied; on the other hand, they demonstrate clearly that there is no influence of thermostresses which could split maxima in these MQW structures at the low-temperature measurements [19]. Since the splitting of electron states caused by tunnelling in ten coupled QW's didn't ap-

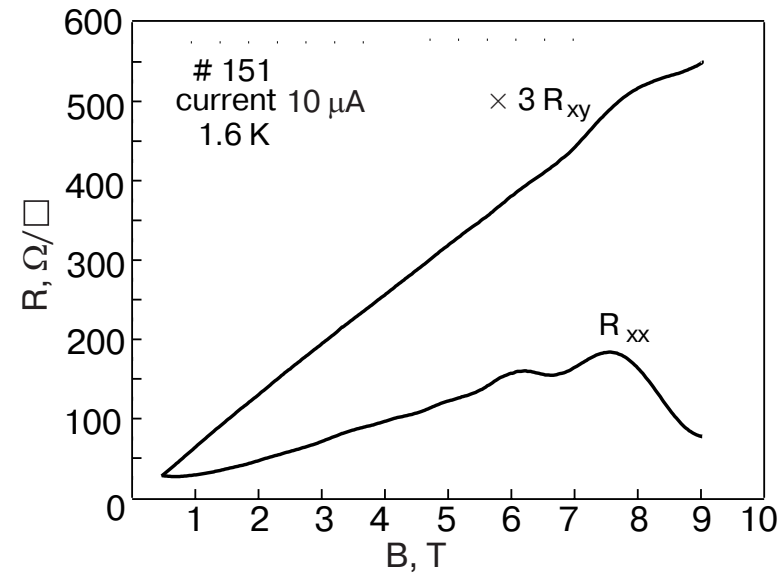

Fig. 7. The records of the $R_{x y}(B)$ and $R_{x x}(B)$ for sample \#151 obtained at low temperature $(T=1.6 \mathrm{~K})$. 
pear at low temperatures $(1.6 \mathrm{~K})$ in the $\mathrm{SdH}$ oscillations and in the IQH effect, the more so they cannot appear at the temperature higher then $77 \mathrm{~K}$.

\subsection{Experimental results: magnetophonon resonance}

The magnetophonon resonance (MPR) appears every time when the phonon frequency $\omega_{L O}$ is equal to the cyclotron frequency $\omega_{c}$ of the electron in a magnetic field, multiplied by the same small integer $M$ :

$$
\omega_{L O}=M \omega_{c},
$$

where $\omega_{c}=e B / m, e$ is electron charge, $m$ is effective mass, $B$ is magnetic field induction.

The resonances are manifested in transverse $\rho_{\mathrm{xx}}$ and longitudinal $\rho_{z z}$ magnetoresistances. The transverse magnetoresistance $\rho_{x x}$ reaches the maximum at resonance values of magnetic fields, which fulfils the specified resonance condition (Eq. (9)). There are two papers know in which MPR was reported examined in superlattices and structures with MQW in the vertical transport (both the magnetic field and the electric current are perpendicular to layers) [20,21]. The present paper reports results of exploring MPR and MQW in the parallel transport. In this instance the measurements can be more sensitive to the strain in layers $[22,23]$.

The MPR researches were performed in pulsed magnetic fields up to $30 \mathrm{~T}$. The pulsed fields were generated by discharging a $10 \mathrm{mF}$ capacitor bank through a copper coil giving a pulse duration of $4 \mathrm{~ms}$. A transient recorder was used to store the transverse magnetoresistance $\rho_{x x}(B)$ and a voltage $\propto \partial B / \partial t$ which was numerically integrated to find the $\mathbf{B}$. Induced voltage in sample leads was compensated for by additional several coils in the field. There was a good agreement between data taken on the rising and falling sides of the pulse for samples with resistances $<300 \mathrm{k} \Omega$. The transverse magnetoresistance was measured between 77 and $340 \mathrm{~K}$ and the MPR oscillations extracted by subtracting a voltage linear in magnetic field. The oscillating part of the magnetoresistance $\Delta \rho_{x x}$ was recorded.

In Fig. 8 the examples of registered curves $\Delta \rho_{x x}(B)$ are shown in which the structure of the observed peaks was faithfully represented because of its astonishing repetition for different MPR harmonics in different temperatures.

The broken curves in Fig. 8, $a$ show the resonance peaks without subtle structures. As the repetition of individual MPR peaks in the given MQW stands out particularly in the magnetic field's interval $0-16 \mathrm{~T}$, where peaks caused by transitions $0-2,0-3,0-4,0-5$ and $0-6$ were observed simultaneously, the resonance curves within this range of induction $B$ of the mag- netic field are represented. Representing them in the whole range of resonance fields, with the transition
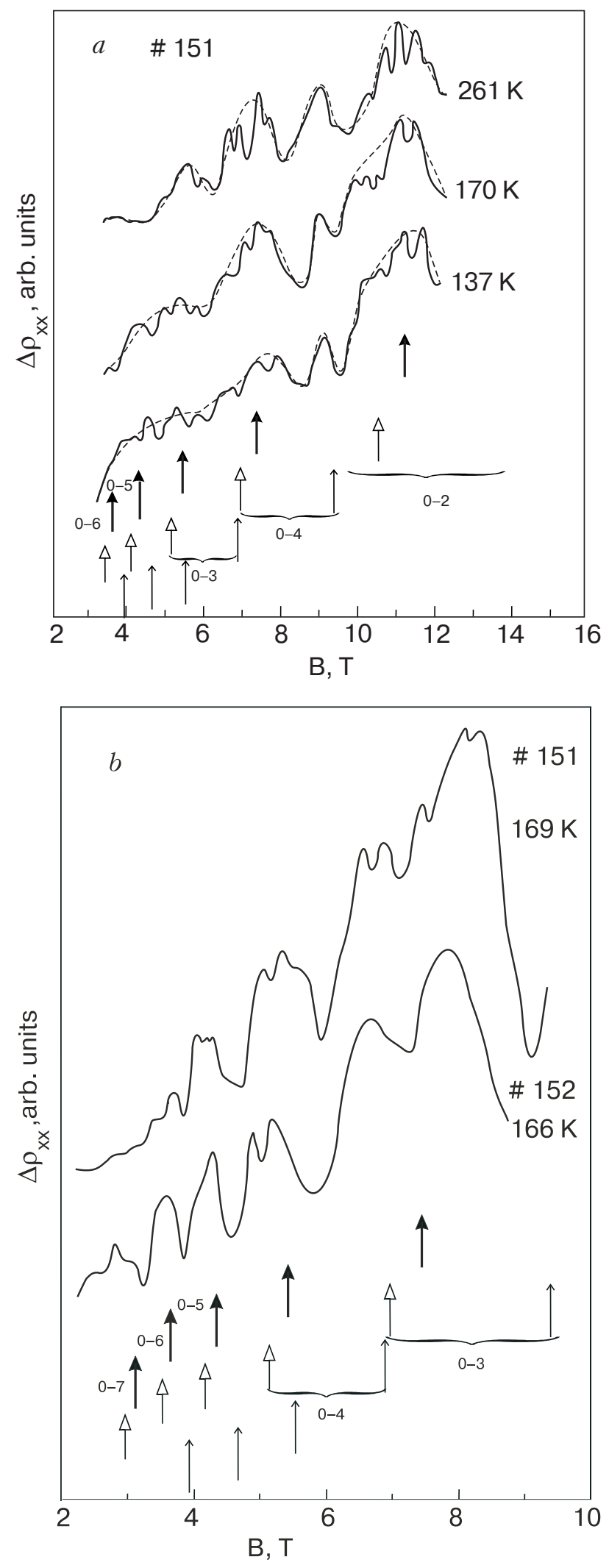

Fig. 8. Experimental recordings of $\Delta \rho_{x x}(B)$ obtained for sample \#151 at three different temperatures higher then $77 \mathrm{~K}(a)$ and samples \#152 $(T=166 \mathrm{~K})$ and \#151 $(T=$ $=169 \mathrm{~K})(b)$. 
0-1 together $(22.5 \mathrm{~T})$ would cause disappearance of interesting structures of peaks and its repetition for different harmonics.

It is necessary to note that:

i) the subtle structure of MPR peaks reappears in each type of MQW, but its character in not the same,

ii) with increasing of temperature the subtle structure became more pronounced.

It follows that the occurrence of the observed peak structure depends neither on the two dimensional density of carriers, nor on the size of the quantum wells but can be connected to phonons.

The thicker arrows in Fig. 8 indicate the position of MPR peaks observed in the single heterostructures $\mathrm{GaAs}^{-} \mathrm{Al}_{x} \mathrm{Ga}_{1-x} \mathrm{As}$ at the temperature $130 \mathrm{~K}$ to facilitate an interpretation of the observed peak structure. In the case of MPR in a single heterostructure each transition between Landau levels corresponds with one peak (structureless) and the resonance fields were somewhat smaller from the theoretical position of MPRs involving the bulk LO phonon of GaAs. The authors [21], where an examination of MPR in the vertical transport in MQW GaAs-AlAs was reported, besides oscillations connected with the absorption of the quantum wells GaAs LO phonons also observed the peaks caused by interaction of electrons with LO phonons of the AlAs barrier. The partly observed peak structure can be explained by the introduction to MPR of two more kinds of barrier phonons. So, the occurrence of a peak at about $9 \mathrm{~T}$ can be explained by a transition $0-3$ of an electron with the absorption AlAs-like LO phonon. The MPR caused by interaction between an electron and phonons from the barrier made by GaAs-like lattice, though, manifest themselves as satellites of each main series transition, on the side of lower magnetic fields. It is mostly visible in Fig. 8,b, where the subtle structure is less prominent.

It is well known that every phonon band in solid solutions of zinc-blend structure consist of four lines [24] which means the existence of the five phonon modes. In case of AlGaAs the average distance between these modes is about $8 \mathrm{~cm}^{-1}$ or $1 \mathrm{meV}$. So, these five modes can generate subtle structure of MPR peaks.

\section{Summary}

The parallel magnetotransport in SQW, DQW and MQW at low temperatures and one higher then $77 \mathrm{~K}$ is studied.

The method of the Landau level energies calculation involving the tunnelling and screening effects in the coupled DQW which enable us to interpret the $\mathrm{SdH}$ oscillations and the IQH effect is developed.

The subtle structure of the MPR peaks in parallel transport of MQW's can not be explained by destruc- tion of the magnetoconductivity caused by thermostresses, because at low temperatures this splitting should manifest itself more strongly, but it does not appear in the SdH oscillations and QHE.

The splitting of electron states caused by tunnelling in coupling ten QW's does not appear at low temperatures $(1.6 \mathrm{~K})$ in $\mathrm{SdH}$ oscillations and QHE, therefore it can not appear at temperature higher then $77 \mathrm{~K}$ when MPR is observed.

There is no doubt that subtle structure of the MPR peaks is connected to phonons.

At the temperatures less than $1 \mathrm{~K}$, the magnetotransport phenomena in a single symmetric $\mathrm{QW}$ of the width greater than $10 \mathrm{~nm}$ reveal the similar features as those characterising the electron transport in DQW.

The $\operatorname{In}_{x} \mathrm{Ga}_{1-x} \mathrm{As} / \mathrm{In}_{x} \mathrm{Al}_{1-x} \mathrm{As}$ (\#1098) structure makes an interesting example of $\mathrm{QW}$ where due to the smooth transition from the barrier to QW the rectangular QW was produced. The calculations confirm that the evaluation of the Landau level energy using rectangular QW model gives better fitting of experimental positions of $\mathrm{SdH}$ peaks then the thriangle QW model. The 2D density of electrons in the channel of the structure in question is very high just like the electron mobility, which is equal to $2.6 \cdot 10^{5} \mathrm{~cm}^{2} /(\mathrm{V} \cdot \mathrm{s})$. Therefore, the proposed engineering of this structure seems to be perspective.

1. W.J. Schaff, P.J. Tasker, M.C. Foisy, and L.F. Eastman, Semiconductors and Semimetals, Academic, New York (1991), vol. 33, Chap. 2.

2. F.H. Pollak, Semiconductors and Semimetals, Academic Press, New York (1990), vol. 32, Chap. 2.

3. Ming-Ta Yang and Yi-Jen Chan, IEEE Trans. El. Dev. 43, 1174 (1996).

4. Y.H. Chen, K.T. Hsu, K.L. Chen, H.H. Lin, and G.J. Jan, Jpn. J. Appl. Phys. 33, 2448 (1994).

5. Y.H. Chen and G.J. Jan, J. Appl. Phys. 77, 6681 (1995).

6. Y.H. Chen and G.J. Jan, IEEE J. Quantum Electron. 33, 574 (1997).

7. G. Tomaka, E.M. Sheregii, T. Kąkol, W. Strupiński, A. Jasik, and R. Jakiela, Physica Status Solidi A195, 127 (2003).

8. G. Tomaka, E.M. Sheregii, T. Kakol, W. Strupiński, R. Jakiela, A. Kolek, A. Stadler, and K. Mleczko, Cryst. Res. Tech. 38, 407 (2003).

9. W. Zawadzki and P. Pfeffer, Proc. NGS 10, IPAP Conf. (2001), Ser. 2, p. 219.

10. W. Zawadzki, J. Phys. C: Solid State Physics 16, 229 (1983).

11. P. Pfeffer and W. Zawadzki, Phys. Rev. B41, 1561 (1990).

12. G.S. Boebinger, H.W. Jiang, L.N. Pfeiffer, and K.W. West, Phys. Rev. Lett. 64, 1793 (1990). 
13. Y.W. Suen, J. Jo, M.B. Santos, L.W. Engel, S.W. Hwang, and M. Shayegan, Phys. Rev. B44, 5947 (1991).

14. T. Ihn, H. Carmona, P.C. Main, L. Eaves, and M. Henini, Phys. Rev. B54, 2315 (1996).

15. T. Jungwith, T.S. Lay, L. Smrcka, and M. Shayegan, Phys. Rev. B56, 1029 (1997)

16. K.M. Brown, N. Turner, J.T. Nicholls, E.H. Linfield, M. Pepper, D.A. Ritchie, and G.A.C. Jones, Phys. Rev. B50, 15465 (1994).

17. M. Kellog, I.B. Spielman, J.P. Eisenstein, L.N. Pfeiffer, and K.W. West, Phys. Rev. Lett. 88, 126804 (2002).

18. D. Huang and M.O. Monosrech, Phys. Rev. B54, 2044 (1996)

19. G. Tomaka, E.M. Sheregii, and T. Kakol, Material Science and Engineering B80, 173 (2001).
20. H. Nogutchi, H. Sakaki, T. Takamasu, and N Miura, Phys. Rev. B45, 12148 (1992).

21. P. Gassot, J. Genoe, D.K. Maude, J.C. Portal, K.S.H. Dalton, D.M. Symons, R.J. Nicholas, F. Arstone, J.F. Palmier, and F. Lauelle, Phys. Rev. B54, 14540 (1996).

22. G. Tomaka, J. Cebulski, E.M. Sheregii, W. Ściuk, W. Strupiriski, and L. Dobrzariski, Acta Phys. Pol. A94, 597 (1998)

23. G. Tomaka, J. Cebulski, E.M. Sheregii, W. Sciuk, W. Strupiński, and I. Dobrzanski, Material Science and Engineering A288, 138 (2000).

24. B.V. Robouch, A. Kisiel, and E.M. Sheregii, Phys. Rev. B64, 073204 (2001). 